Article

\title{
Responses of Different Panicum miliaceum L. Genotypes to Saline and Water Stress in a Marginal Mediterranean Environment
}

\author{
Caterina Caruso ${ }^{1}$, Carmelo Maucieri ${ }^{1, *}$, Andrea Berruti ${ }^{2}$, Maurizio Borin ${ }^{1}$ and \\ Antonio Carlo Barbera ${ }^{3,4}$ \\ 1 Department of Agronomy, Food, Natural Resources, Animals and Environment-DAFNAE, \\ University of Padua, Agripolis Campus, Viale dell'Università 16, 35020 Legnaro, Italy; \\ ca.ty87@hotmail.it (C.C.); maurizio.borin@unipd.it (M.B.) \\ 2 Institute for Sustainable Plant Protection-Turin UOS, National Research Council, Viale Mattioli 25, \\ 10125 Torino, Italy; andrea.berruti@unito.it \\ 3 Department of Agriculture, Food and Environment (Di3A), University of Catania, Via Valdisavoia 5, \\ 95123 Catania, Italy; ac.barbera@unict.it \\ 4 Trees and Timber Institute (IVALSA), Consiglio Nazionale delle Ricerche (CNR) Via Paolo Gaifami 18, \\ 95126 Catania, Italy \\ * Correspondence: carmelo.maucieri@unipd.it; Tel.: +39-049-827-2859
}

Received: 25 November 2017; Accepted: 10 January 2018; Published: 18 January 2018

\begin{abstract}
The aims of this study were to evaluate: (1) the effect of sodium chloride $(\mathrm{NaCl})$ and mannitol at different osmotic pressures on the germination of three proso millet (Panicum miliaceum L.) genotypes (VIR 9181, Unikum, and Kinelskoje) under controlled laboratory conditions; and (2) the effects of irrigation water salinity, maximum crop evapotranspiration (ETm) restitution regimes, and arbuscular mycorrhizal fungi (AMF) inoculation on forage production in a marginal Mediterranean soil for the genotypes that showed the highest and lowest seed germination. In the laboratory experiment, the Unikum genotype showed the highest seed germination $(95.1 \%)$, whereas the lowest was found for Kinelskoje (80.4\%). Regardless of the osmoticum type, germination was significantly reduced by osmotic pressure increases. Unikum showed a higher fresh biomass yield (FBY) $\left(620.4 \pm 126.3 \mathrm{~g} \mathrm{~m}^{-2}\right)$ than Kinelskoje $\left(340.0 \pm 73.5 \mathrm{~g} \mathrm{~m}^{-2}\right)$. AMF inoculation did not influence FBY under salt conditions, while in the absence of salt conditions it significantly increased the Unikum FBY (+50.7\%) as compared to the uninoculated treatment $\left(552.5 \pm 269 \mathrm{~g} \mathrm{~m}^{-2}\right)$. The $25 \%$ ETm significantly reduced FBY in both genotypes $(-86.2 \%$ and $-84.1 \%$ for Unikum and Kinelskoje, respectively) sd compared to the $100 \%$ ETm treatments $\left(1090.3 \pm 49.7 \mathrm{~g} \mathrm{~m}^{-2}\right.$ in Unikum and $587 \pm 72.2 \mathrm{~g} \mathrm{~m}^{-2}$ in Kinelskoje). The obtained results give novel information about proso millet forage production in low-input agriculture in marginal semi-arid Mediterranean land.
\end{abstract}

Keywords: Rhizophagus intraradices; Funneliformis mosseae; sodium chloride; bio-fertilizer; proso millet

\section{Introduction}

In semi-arid and arid regions, salinity is one of the major causes of land degradation [1,2] and leads to huge economic losses due to the reduction in total arable land area and crop productivity [3]. Salts effects on agricultural soils are generally tied to soluble minerals present in irrigation water and high fertilization input [4,5]. In Europe, about 3.8 million ha are affected by soil salinization due to natural saline soils in Spain, Hungary, Greece, and Bulgaria, or irrigation with saline water in significant areas of Sicily and the Ebro Valley in Spain and in other smaller parts of Italy, Hungary, Greece, Portugal, France, Slovakia, and Romania [6]. Plants growing in saline soil are subjected to three distinct physiological stresses: (1) toxic effects of specific ions such as sodium and chloride, 
which disrupt the structure of enzymes and other macromolecules, damage cell organelles, reduce photosynthesis and respiration, inhibit protein synthesis, and induce ion deficiency $[7,8] ;(2)$ osmotic effects due to physiological drought because plants must maintain lower internal osmotic potential to prevent water from moving from the roots into the soil [9]; and (3) nutrient imbalances caused by depression in uptake and/or transport [10,11].

Among a wide array of rhizosphere microorganisms, arbuscular mycorrhizal fungi (AMF) are an essential component of sustainable and low-input agricultural systems [12]. AMF symbiosis can exert positive effects on crop production [13-16] and improve plant tolerance to abiotic stresses [17-21] such as drought and salinity. Possible AMF-mediated adaptation mechanisms inducing plant tolerance to saline conditions [22] include: (1) nutrient uptake improvement, especially phosphorus (P) [23-25]; (2) accumulation of soluble sugars into the roots [26]; (3) $\mathrm{K}^{+} / \mathrm{Na}^{+}$ratio adjustment $[27,28]$; and (4) antioxidant enzymatic activities [29]. Although AMF can be found in saline soils, some of their features, such as spore germination, fungal hyphae growth [20], formation of mycorrhizal arbuscules [30,31], and root colonization levels [32] may also be negatively affected by high salinity. Despite these mycorrhizal behaviors, the positive influence on crop production exerted by AMF inoculum under salinity stress conditions is not yet fully understood.

Proso millet (Panicum miliaceum L.) is a C4 annual plant, which moved from Eastern and Central Asia to Europe about 3000 years ago [33]. It is a mycorrhizal plant that has previously been reported to strongly benefit from AMF inoculation [34]. Moreover, proso millet is the world's sixth most important cereal grain [35] and it is mainly cultivated in Africa and Asia [36]. In Europe, it is cultivated in $61,233 \mathrm{ha}$, with an average grain yield of $1.9 \mathrm{Mg} \mathrm{ha}^{-1}$ [37]. This crop has many desirable agronomic traits, including a short growing season (60-90 days), and low nutrient and water requirements, as well as excellent tolerance to salt, drought, high temperature, and other extreme conditions [38]. For these reasons, proso millet can be also cultivated in marginal lands where other cereals do not fully succeed [39]. In several countries, it is harvested primarily for human consumption [40], but it is also used as fodder [41]. However, specific scientific literature on proso millet used as a forage crop, especially in the Mediterranean area, is lacking. In Iran, fresh biomass yields ranging from 16.1 $\mathrm{Mg} \mathrm{ha}^{-1}$ [42] to $43.6 \mathrm{Mg} \mathrm{ha}^{-1}$ [43] have been reported.

The semi-arid zones of Southern Italy are particularly prone to erosion due to a combination of climatic and edaphic factors, including soil salinity. In Sicily, marginal land reclamation programs are being now evaluated, and sustainable practices, such as proso millet cultivation with AMF biofertilization, could represent a valid option.

The aims of this study on proso millet were to evaluate: (1) in laboratory conditions, the effect of osmo-salinity stress on seed germination of different genotypes; and (2) in a marginal Mediterranean soil, the effect of AMF inoculation with different saline water levels and crop evapotranspiration restitutions (ETm) on the milk-dough fresh biomass yield (FBY) for forage production.

\section{Materials and Methods}

\subsection{Laboratory Experiment}

The laboratory experiments were carried out at the Department of Agriculture, Food and Environment of the University of Catania (Italy). Three different genotypes of Panicum miliaceum L. (VIR 9181, Unikum, and Kinelskoje) were chosen for seed imbibition and germination tests. The used seed lots had 1000-seed weights of $4.85 \mathrm{~g}$ for VIR 9181, $5.63 \mathrm{~g}$ for Unikum, and $6.60 \mathrm{~g}$ for Kinelskoje. The studied treatments are listed in Table 1.

Salinity stress was induced by adding $\mathrm{NaCl}$ at concentrations able to give the same osmotic potentials as the mannitol solutions. Osmotic potential in both solutions was verified using an automatic cryoscopic osmometer (Gonotec Osmomat 030 model, Berlin, Germany). 
Table 1. Proso millet fresh weight yield components of main culm yield.

\begin{tabular}{cccc}
\hline Treatment Number & Osmotic Agent & Osmotic Agent Weight $\mathbf{~ g ~ L ~}^{-\mathbf{1}}$ ) & Osmotic Pressure (MPa) \\
\hline 1 & Distilled water & 0 & 0 \\
2 & $\mathrm{NaCl}$ & 4.1 & -0.250 \\
3 & $\mathrm{NaCl}$ & 7.6 & -0.500 \\
4 & $\mathrm{NaCl}$ & 11.1 & -0.750 \\
5 & Mannitol & 21.6 & -0.250 \\
6 & Mannitol & 42.5 & -0.500 \\
7 & Mannitol & 63.4 & -0.750 \\
\hline
\end{tabular}

\subsubsection{Seed Water Uptake}

For each treatment, seed water uptake at $2,4,17$, and $21 \mathrm{~h}$ of imbibition, was measured. For this purpose, 30 millet seeds of uniform size were hand-selected for each genotype and placed in Petri dishes $(\varnothing 9 \mathrm{~cm})$ with $9 \mathrm{~mL}$ of each studied solution under dark conditions at a temperature of $25 \pm 1{ }^{\circ} \mathrm{C}$. Each treatment was replicated three times. After initiation, millet seeds were removed at each measuring time, drained, blotted with absorbent paper, weighed and placed again into the Petri dishes. Percentage seed water uptake was determined as:

Seed water uptake $(\%)=[($ final weight - initial weight $) /$ initial weight $] \times 100$

\subsubsection{Seed Germination}

Hand-selected seeds of uniform size were surface sterilized with $5 \%(w / v)$ calcium hypochlorite for $5 \mathrm{~min}$ and rinsed four times with deionized water. These seeds were then transferred to Petri dishes (20 seeds per $\varnothing 9 \mathrm{~cm}$ Petri dish) containing one Whatman ${ }^{\circledR}$ Filter Paper moistened with $9 \mathrm{~mL}$ of the studied solutions. Each treatment was replicated three times. Petri dishes were tightly sealed with Parafilm ${ }^{\circledR}$ to avoid water depletion. Seeds were allowed to germinate in a growth chamber at a temperature of $25 \pm 1{ }^{\circ} \mathrm{C}$ in the dark. Seeds were scored as germinated when a radicle extrusion $\geq 2 \mathrm{~mm}$ long was observed. Germination percentage (GP) was calculated according to the International Seed Testing Association (ISTA) method [44]:

$$
\mathrm{GP}=\text { Number of normally germinated seeds/total seeds number } \times 100
$$

Moreover, seedling vigor index (SVI) was calculated by the following formula according to Ali and Idris [45]:

$$
\text { SVI }=(\text { seedling length }(\mathrm{cm}) \times \text { germination percentage }) / 100
$$

\subsection{Field Experiment}

The two millet genotypes that showed the highest (Unikum) and lowest (Kinealskoje) germination percentages in a preliminary laboratory trial were evaluated in an open field study (summer 2014) to test genotype-specific response under stress conditions. The experiment was conducted at the Experimental Farm "Cibali" of the Istituto Agrario Siciliano Valdisavoia $\left(37^{\circ} 31^{\prime} \mathrm{N}, 15^{\circ} 04^{\prime} \mathrm{E}, 84 \mathrm{~m}\right.$ a.s.l.) in Catania (Italy) in volcanic soil with sandy texture, electrical conductivity of $1.9 \mathrm{dS} \mathrm{m}^{-1}$, and $\mathrm{pH}$ 6.3. Over the short experimental period the main daily meteorological variables of maximum, minimum, and average temperature, rainfall, solar radiation, and potential evapotranspiration $\left(\mathrm{ET}_{0}\right)$ were provided by the INAF (Catania Astrophysical Observatory), from agrometeorological stations located approximately $500 \mathrm{~m}$ from the experimental site (Figure 1a,b). 

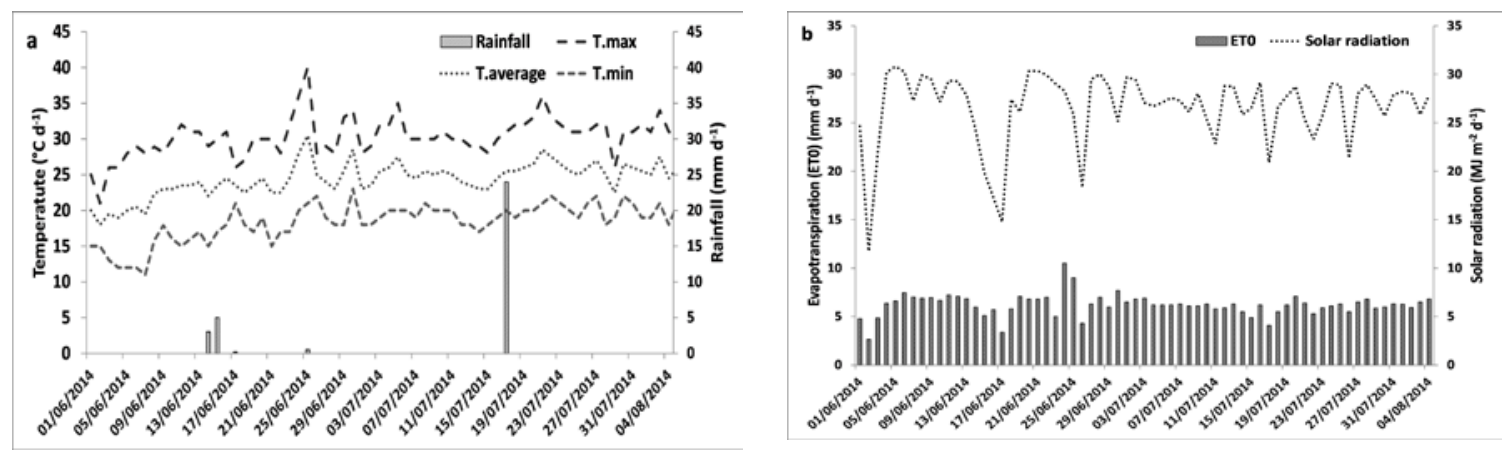

Figure 1. Environmental site conditions. (a) Rainfall and minimum (T.min), maximum (T.max) and average (T.average) temperature; and $(\mathbf{b})$ potential evapotranspiration $\left(\mathrm{ET}_{0}\right)$ and solar radiation.

A split-plot design with plots of $2 \mathrm{~m} \times 1 \mathrm{~m}$ replicated two times was adopted. AMF inoculation was the main factor (AMF-Y = inoculated plots and AMF-N = not inoculated plots). Sub-plots involved salt stress with $\mathrm{NaCl}$ in irrigation water (without $\mathrm{NaCl}\left(0.5 \mathrm{dS} \mathrm{m}^{-1}\right)=$ Salt- $\mathrm{N}$ and with $\mathrm{NaCl}$ at $5 \mathrm{dS} \mathrm{m}^{-1}=$ Salt-Y), restitution of crop evapotranspirated water ( $25 \%$ ETm and $\left.100 \% \mathrm{ETm}\right)$, and millet genotypes ( $\mathrm{U}=$ Unikum; $\mathrm{K}=$ Kinelskoje). Maximum crop evapotranspiration (ETm) was calculated in agreement with Food and Agriculture Organization of the United Nations "Irrigation and Drainage Paper 56" [45] through the reference evapotranspiration $\left(\mathrm{ET}_{0}\right)$ and crop coefficient $(\mathrm{Kc})$.

Sowing, weeding and thinning operations were done manually. The two genotypes were sowed on 10 June 2014, with 360 seeds $\mathrm{m}^{-2}$, adopting an inter-row distance of $15 \mathrm{~cm}$ and a sowing depth of about $2 \mathrm{~cm}$. During the sowing, AMF inoculation (based on Rhizophagus intraradices, Funneliformis mosseae, Glomus spp., and other microorganisms) was manually carried out at 600 propagules $\mathrm{m}^{-2}$, distributing commercial inoculum (AEGIS SYM PASTIGLIA) along the row. All plots, for the first eight days after sowing (DAS), received the same water volume (about $6 \mathrm{~mm}$ of fresh water every two days) for seed germination. Subsequently, at 10 DAS when the crop had the first two leaves fully expanded, water restitution and $\mathrm{NaCl}$ treatments took place until harvest, distributing a total of $30.6 \mathrm{~mm}$ for $25 \%$ ETm and $159.3 \mathrm{~mm}$ for $100 \% \mathrm{ETm}$. After millet plants emerged, eight randomly selected plants per treatment were marked and were monitored weekly for culm height, leaf number, and phenological phase, in agreement with the Biologische Bundesantalt, Bundessortenamt and CHemische Industrie (BBCH) scale [46]. Moreover, the SPAD (Soil and Plant Analyzer Development) index, an indirect measure of chlorophyll content, was measured three times from 34 to 48 DAS (Minolta SPAD-502, Konica-Minolta, Osaka, Japan).

On 4 August 2014, millet was harvested at the milk-dough stage, and fresh biomass yield and its components were detected. In four plants per subplot, the leaf area surface was measured using WinDias 2.0 (ODELTA-T Devices Ltd. 1995-2000, Cambridge, UK).

\subsection{Statistical Analysis}

Data were analyzed by ANOVA in order to evaluate the treatment effects. A post hoc test was performed to compare means using Fisher's Least Significant Difference (LSD) test $(\alpha=0.05)$. An arcsine transformation was applied to all data expressed as a percentage before performing ANOVA.

\section{Results}

\subsection{Laboratory Experiment}

\subsubsection{Seed Water Uptake}

Seed water uptake dynamics during the first $21 \mathrm{~h}$ of imbibition were significantly $(p<0.001)$ influenced by the osmotic pressure. Regardless of the genotype, significantly higher water uptake was 
measured after 4, 17, and $21 \mathrm{~h}$ in the control (0.0 MPa) as compared to other osmotic pressure levels (Figure 2a). Considering genotypes, after 2 and $4 \mathrm{~h}$, the water uptake in Kinelskoje was significantly higher $(p<0.001)$ than in the other two genotypes, whereas no differences were found after 17 and $21 \mathrm{~h}$ (Figure 2b).
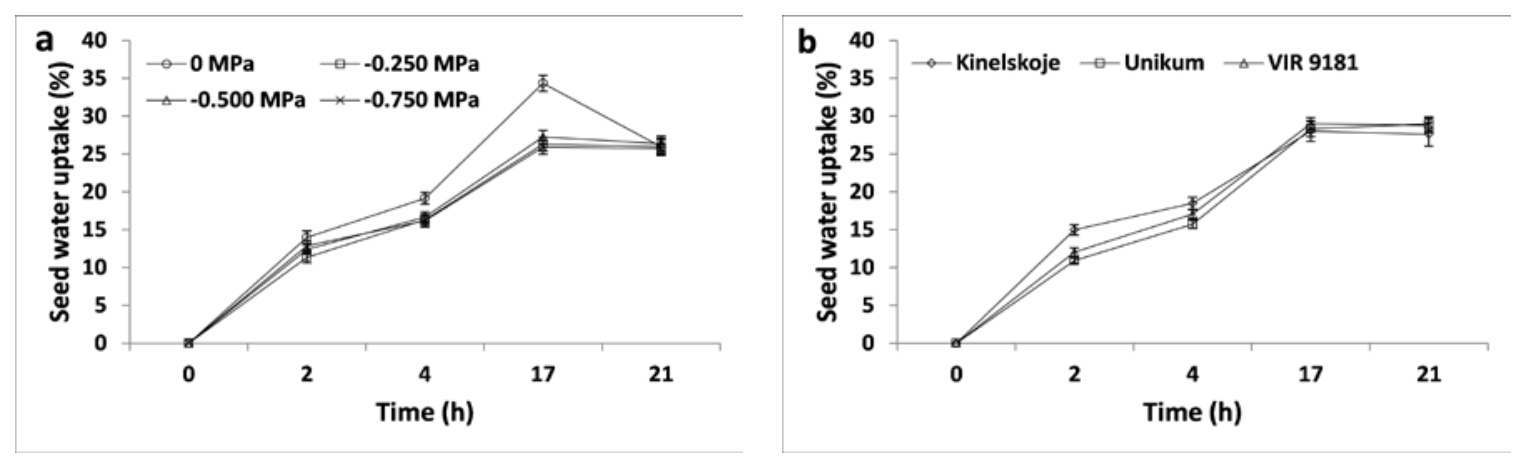

Figure 2. Time course of water uptake as affected by (a) osmotic pressure and (b) millet genotypes.

At $21 \mathrm{~h}$ from the start of seed imbibition, water uptake was significantly $(p<0.05)$ affected by the interaction between millet genotypes and osmotic pressure (Figure 3a). Kinelskoje presented the highest water uptake at $0.0 \mathrm{MPa}(38.7 \%)$ but was the most sensitive among the three genotypes to the decrease of water potential from $-0.250 \mathrm{MPa}$ to $-0.500 \mathrm{MPa}$. At the lowest water potential $(-0.750 \mathrm{MPa})$, all genotypes showed the same water uptake (on average $25.7 \%$ ).
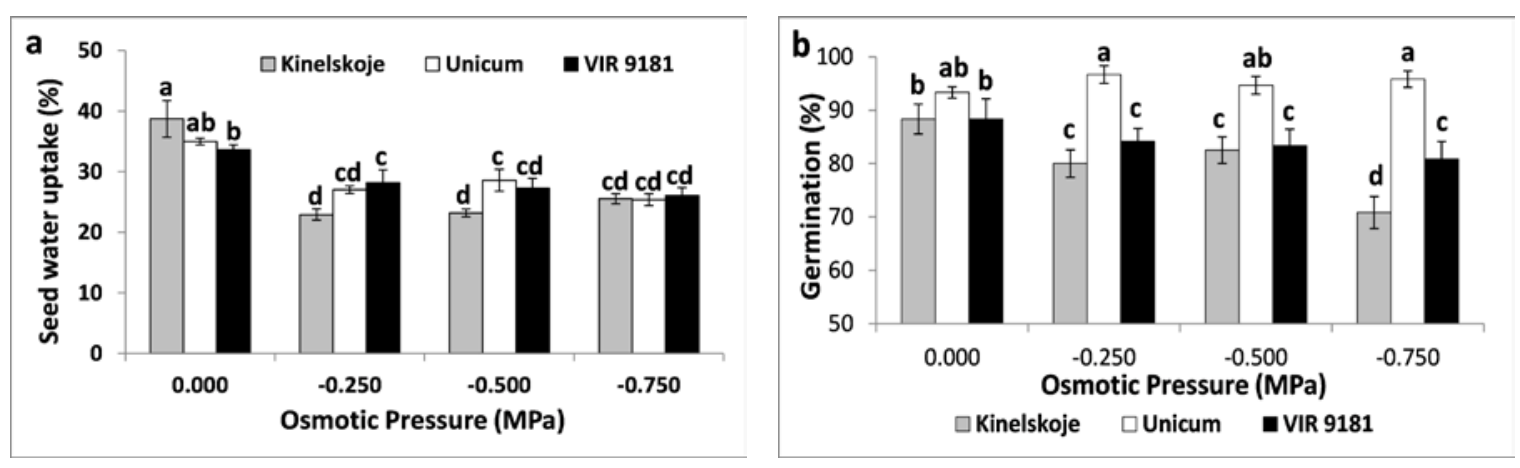

Figure 3. (a) Water uptake at $21 \mathrm{~h}$ and (b) germination percentage at $96 \mathrm{~h}$ of millet genotypes under osmotic pressure. Different letters show statistical differences of the treatments at $p<0.05$ (Least Significant Difference-Fisher test).

\subsubsection{Seed Germination}

The germination percentages of VIR 9181 and Kinelskoje were negatively affected by the decrease in water potential. In VIR 9181, significant differences in seed germination were observed from 0.0 to $-0.250 \mathrm{MPa}$, with a decrease of $-4.72 \%$, while no differences from -0.250 to $-0.750 \mathrm{MPa}$ were found (Figure $3 b)$. In Kinelskoje, significantly $(p<0.05)$ lower germination values were observed at -0.250 and $-0.500 \mathrm{MPa}$ (on average $-8.0 \%$ ), and at $-0.750 \mathrm{MPa}$ (on average $-19.8 \%$ ), compared to water control (Figure 3b). Unikum seed germination was not affected by osmotic pressure, showing the highest germination $(95.1 \% \pm 0.75)$ (Figure $3 b)$ and seedling vigor index (SVI) $(3.65 \pm 0.30)$ values (Table 2). In addition to water uptake and germination rate, low water potential ( $-0.750 \mathrm{MPa})$ was the most detrimental factor for seedling growth and, regardless of the treatment applied, resulted in the strongest significant $(p<0.01)$ decrease of SVI $(-70.9 \%)$ compared to the control (Table 2$)$. 
No differences were found in seed water uptake, germination, and seedling vigor index in relation to the osmoticum ( $\mathrm{NaCl}$ and mannitol).

Table 2. Seedling vigor index (SVI) of millet genotypes in relation to osmotic pressure.

\begin{tabular}{|c|c|c|c|c|c|c|c|c|}
\hline \multirow{2}{*}{$\begin{array}{c}\text { Osmotic } \\
\text { Pressure (MPa) }\end{array}$} & \multicolumn{6}{|c|}{ Genotypes } & \multirow{2}{*}{\multicolumn{2}{|c|}{ Mean }} \\
\hline & \multicolumn{2}{|c|}{ Kinelskoje } & \multicolumn{2}{|c|}{ Unikum } & \multicolumn{2}{|c|}{ VIR 9181} & & \\
\hline 0.0 & \multicolumn{2}{|c|}{$3.93 \pm 0.17$} & \multicolumn{2}{|c|}{$5.48 \pm 0.07$} & \multicolumn{2}{|c|}{$5.35 \pm 0.16$} & \multicolumn{2}{|c|}{$4.92 \pm 0.19$} \\
\hline-0.250 & \multicolumn{2}{|c|}{$2.48 \pm 0.29$} & \multicolumn{2}{|c|}{$4.08 \pm 0.46$} & \multicolumn{2}{|c|}{$2.66 \pm 0.23$} & \multicolumn{2}{|c|}{$3.07 \pm 0.25$} \\
\hline-0.500 & \multicolumn{2}{|c|}{$2.45 \pm 0.32$} & \multicolumn{2}{|c|}{$3.01 \pm 0.36$} & \multicolumn{2}{|c|}{$2.93 \pm 0.36$} & \multicolumn{2}{|c|}{$2.79 \pm 0.20$} \\
\hline-0.750 & \multicolumn{2}{|c|}{$1.08 \pm 0.13$} & \multicolumn{2}{|c|}{$2.04 \pm 2.04$} & \multicolumn{2}{|c|}{$1.18 \pm 0.10$} & \multicolumn{2}{|c|}{$1.43 \pm 0.13$} \\
\hline Mean & \multicolumn{2}{|c|}{$2.48 \pm 0.24$} & \multicolumn{2}{|c|}{$3.65 \pm 0.30$} & \multicolumn{2}{|c|}{$3.03 \pm 0.33$} & 3.05 & 0.18 \\
\hline \multirow{2}{*}{ ANOVA } & \multirow{2}{*}{ SS } & \multirow{2}{*}{ DF } & \multirow{2}{*}{ MS } & \multirow[b]{2}{*}{$\mathbf{F}$} & \multirow{2}{*}{ Prob. F } & \multirow{2}{*}{ Sign. F } & \multicolumn{2}{|c|}{ LSD } \\
\hline & & & & & & & $p<0.05$ & $p<0.01$ \\
\hline G & 15.7309 & 2 & 7.8654 & 17.2022 & $2.33 \times 10^{-6}$ & $* *$ & 0.39247 & 0.52356 \\
\hline OP & 108.764 & 3 & 36.256 & 79.2911 & $1.30 \times 10^{-18}$ & $* *$ & 0.45319 & 0.60456 \\
\hline $\mathrm{S}$ & 0.0147 & 1 & 0.0147 & 0.0321 & $8.59 \times 10^{-1}$ & & 0.32045 & 0.42749 \\
\hline $\mathrm{G} \times \mathrm{OP}$ & 6.1833 & 6 & 1.0306 & 2.2539 & $5.37 \times 10^{-2}$ & & 0.78495 & 1.04713 \\
\hline$G \times S$ & 0.3383 & 2 & 0.1692 & 0.3700 & $6.93 \times 10^{-1}$ & & 0.55504 & 0.74043 \\
\hline $\mathrm{OP} \times \mathrm{S}$ & 0.5102 & 3 & 0.1701 & 0.3719 & $7.74 \times 10^{-1}$ & & 0.64091 & 0.85498 \\
\hline $\mathrm{G} \times \mathrm{OP} \times \mathrm{S}$ & 4.1026 & 6 & 0.6838 & 1.4954 & $2.00 \times 10^{-1}$ & & 1.11009 & 1.48086 \\
\hline Residual & 21.9472 & 48 & 0.4572 & & & & & \\
\hline Total & 157.5909 & 71 & & & & & & \\
\hline
\end{tabular}

$\mathrm{G}=$ genotype; $\mathrm{OP}=$ osmotic pressure; $\mathrm{S}=$ solute $(\mathrm{NaCl}$ and mannitol). $\mathrm{SS}=, \mathrm{DF}=, \mathrm{MS}=, \mathrm{F}=$, Prob. $\mathrm{F}=$, Sign. $\mathrm{F}=$, LSD $=$ Least Significant Difference; ${ }^{* *} p<0.01$.

\subsection{Field Experiment}

\subsubsection{Fresh Biomass Yield (FBY)}

FBY was significantly influenced by all the studied factors. With respect to the average of the other studied factors, Unikum showed a significantly higher FBY $(+82.5 \%)$ than Kinelskoje $\left(340.0 \pm 73.5 \mathrm{~g} \mathrm{~m}^{-2}\right)$. AMF-inoculated plots (AMF-Y) showed a greater FBY $(+19.4 \%)$ than uninoculated plots (AMF-N) $\left(437.7 \pm 112.3 \mathrm{~g} \mathrm{~m}^{-2}\right)$. The lowest ETm restitution $(25 \%)$ resulted in a significant decrease $(-85.5 \%)$ in FBY as compared to $100 \%$ ETm $\left(838.6 \pm 77.5 \mathrm{~g} \mathrm{~m}^{-2}\right)$. Irrigation with saline water significantly reduced (-28.6\%) FBY as compared to the absence of salt stress $\left(560.1 \pm 116.1 \mathrm{~g} \mathrm{~m}^{-2}\right)$.

Under salt stress conditions, AMF inoculation did not exert significant effects on FBY in either of the millet genotypes (Figure 4). In the treatment without $\mathrm{NaCl}$ addition in irrigation water (Salt-N), only in Unikum did the AMF-Y treatment result in a significant $(p<0.01) \mathrm{FBY}$ increase $(+50.7 \%)$, as compared to the AMF-N treatment $\left(552.5 \pm 269.0 \mathrm{~g} \mathrm{~m}^{-2}\right)$.

Considering water restitution, at $100 \% \mathrm{ETm}$, the treatment with $\mathrm{NaCl}$ addition in irrigation water (Salt-Y) resulted in a significant $(p<0.01)$ FBY decrease in both Unikum $(-13.6 \%)$ and Kinelskoje $(-45.9 \%)$, as compared to the Salt- $\mathrm{N}$ treatments $\left(1169.8 \mathrm{~g} \mathrm{~m}^{-2}\right.$ in Unikum and $761.9 \mathrm{~g} \mathrm{~m}^{-2}$ in Kinelskoje). At 25\% ETm in Unikum, the Salt-Y treatment resulted in a significant $(p<0.01)$ reduction $(-60.3 \%)$ of FBY as compared to the Salt-N treatment $\left(215.4 \pm 100.6 \mathrm{~g} \mathrm{~m}^{-2}\right)$, whereas in Kinelskoje, no statistical difference was observed between salt treatments, with a mean value of $252 \pm 63.8 \mathrm{~g} \mathrm{~m}^{-2}$. The $25 \%$ ETm significantly $(p<0.05)$ reduced FBY in both genotypes $(-86.2 \%$ and $-84.1 \%$ for Unikum and Kinelskoje, respectively) compared to the $100 \%$ ETm treatments $\left(1090.3 \pm 49.7 \mathrm{~g} \mathrm{~m}^{-2}\right.$ in Unikum and $587 \pm 72.2 \mathrm{~g} \mathrm{~m}^{-2}$ in Kinelskoje). The AMF inoculation at $100 \%$ ETm restitution did not significantly affect the FBY compared to the uninoculated treatment (grand mean $838.6 \pm 112.4 \mathrm{~g} \mathrm{~m}^{-2}$ ). Conversely, at $25 \%$ ETm, a significant $(p<0.01)$ increase of FBY was observed in AMF-inoculated plants (Figure 5). 


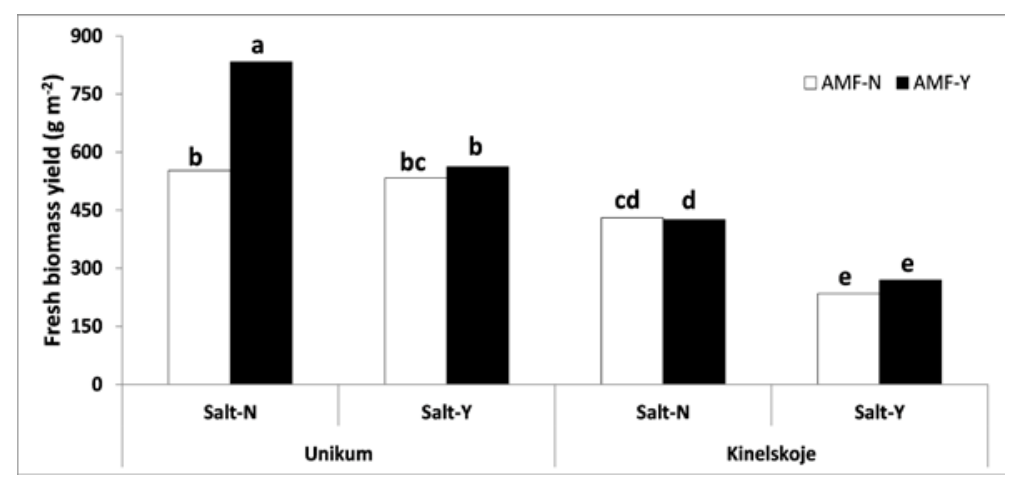

Figure 4. Effect of arbuscular mycorrhizal inoculation on fresh biomass yield in two millet genotypes under salt conditions. Different letters show statistical differences of the treatments at $p<0.01$ (Least Significant Difference-Fisher test). AMF-Y = AMF-inoculated treatment; AMF-N = AMF uninoculated treatment; Salt- $\mathrm{Y}=$ treatment with $\mathrm{NaCl}$ addition in irrigation water; Salt- $\mathrm{N}$ = treatment without $\mathrm{NaCl}$ addition in irrigation water.

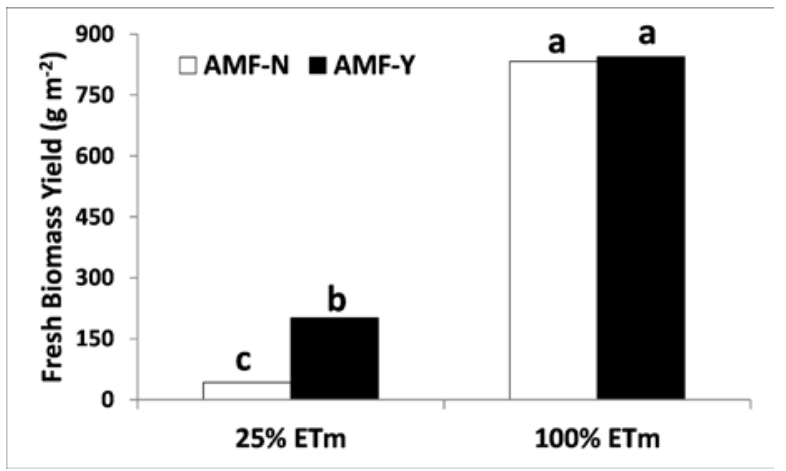

Figure 5. Effect of arbuscular mycorrhizal inoculation on fresh biomass yield under different water restitution levels. Different letters show statistical differences of the treatments at $p<0.01$ (Least Significant Difference-Fisher test). AMF-Y = AMF-inoculated treatment; AMF-N = AMF uninoculated treatment; $25 \% \mathrm{ETm}$ = restitution of crop evapotranspirated water at $25 \% ; 100 \% \mathrm{ETm}=$ restitution of crop evapotranspirated water at $100 \%$.

At harvest time, the relative moisture of FBY ranged from 70.3\% (25\% ETm, AMF-Y, Salt-N) to 88.2\% (100\% ETm, AMF-N, Salt-Y) in Kinelskoje and from 74.9\% (25\% ETm, AMF-N, Salt-N) to 89.3\% $(100 \%$ ETm, AMF-Y, Salt-N) in Unikum.

\subsubsection{Shoot Density}

Shoot density was significantly $(p<0.01)$ affected by genotype and water restitution. Comparing the two genotypes, Unikum showed a higher shoot density (+35\%) than Kinelskoje $\left(131 \pm 7.3\right.$ plants $\mathrm{m}^{-2}$ ). Regardless of the genotype and AMF inoculation treatments, 25\% ETm restitution resulted in a significant $(p<0.001)$ decrease $(-25.5 \%)$ in shoot density compared to $100 \%$ ETm restitution $\left(176 \pm 13\right.$ plants $\left.\mathrm{m}^{-2}\right)$. The significant interaction $(p<0.01)$ between water restitution and AMF inoculation showed a positive effect of AMF inoculum at the lower ETm restitution and a negative one at full ETm restitution (Figure 6). 


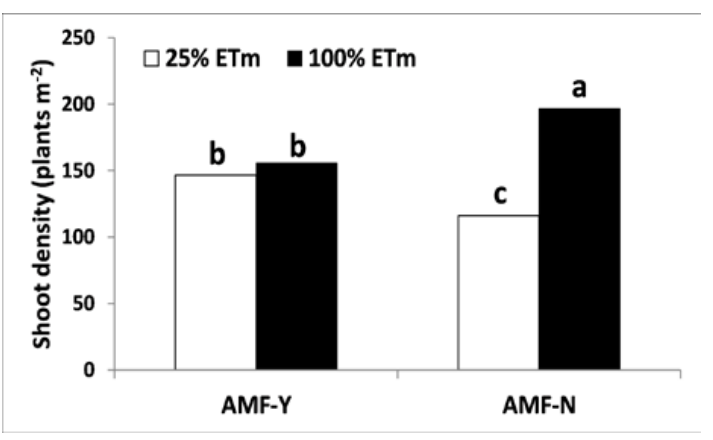

Figure 6. Effect of arbuscular mycorrhizal inoculation on shoot density under different water restitution levels. Different letters show statistical differences of the treatments at $p<0.01$ (Least Significant Difference-Fisher test). AMF-Y = AMF-inoculated treatment; AMF-N = AMF uninoculated treatment; $25 \% \mathrm{ETm}=$ restitution of crop evapotranspirated water at $25 \% ; 100 \% \mathrm{ETm}=$ restitution of crop evapotranspirated water at $100 \%$.

\subsubsection{Culm Height and Fresh Culm Weight}

The treatment effect on culm height is reported in Table 3.

Table 3. Bio-morphological proso millet characteristics. ETm: maximum crop evapotranspiration.

\begin{tabular}{|c|c|c|c|c|c|c|c|c|c|}
\hline \multicolumn{4}{|c|}{ Treatments } & \multicolumn{2}{|c|}{$\begin{array}{l}\text { Culm Height } \\
\text { (cm) }\end{array}$} & \multicolumn{2}{|c|}{$\begin{array}{c}\text { Panicle } \\
\text { Length }(\mathrm{cm})\end{array}$} & \multicolumn{2}{|c|}{$\begin{array}{l}\text { Leaf Number } \\
\left(\text { plant }^{-1}\right)\end{array}$} \\
\hline \multirow{7}{*}{ Kinelskoje } & \multirow{3}{*}{ AMF-Y } & Salt-Y & $\begin{array}{c}25 \% \text { ETm } \\
100 \% \text { ETm }\end{array}$ & \multicolumn{2}{|c|}{$\begin{array}{l}13.3 \pm 2.37 \\
25.1 \pm 2.09\end{array}$} & \multicolumn{2}{|c|}{$\begin{array}{l}2.06 \pm 1.35 \\
10.6 \pm 1.11\end{array}$} & \multicolumn{2}{|c|}{$\begin{array}{l}3.75 \pm 0.45 \\
5.50 \pm 0.33\end{array}$} \\
\hline & & \multirow[b]{2}{*}{ Salt-N } & $25 \%$ FTm & \multirow{2}{*}{\multicolumn{2}{|c|}{$\begin{array}{l}18.1 \pm 3.59 \\
42.8 \pm 2.27\end{array}$}} & \multirow{2}{*}{\multicolumn{2}{|c|}{$\begin{array}{l}11.2 \pm 0.64 \\
13.7 \pm 0.39\end{array}$}} & \multirow{2}{*}{\multicolumn{2}{|c|}{$\begin{array}{l}4.88 \pm 0.23 \\
4.50 \pm 0.46\end{array}$}} \\
\hline & & & $100 \%$ ETm & & & & & & \\
\hline & \multirow{4}{*}{ AMF-N } & \multirow{2}{*}{ Salt-Y } & $25 \%$ ETm & \multirow{2}{*}{\multicolumn{2}{|c|}{$\begin{array}{l}12.1 \pm 1.43 \\
16.2 \pm 1.02\end{array}$}} & \multirow{2}{*}{\multicolumn{2}{|c|}{$\begin{array}{l}0.00 \pm 0.00 \\
10.9 \pm 0.33\end{array}$}} & \multirow{2}{*}{\multicolumn{2}{|c|}{$\begin{array}{l}3.38 \pm 0.26 \\
5.00 \pm 0.19\end{array}$}} \\
\hline & & & $100 \%$ ETm & & & & & & \\
\hline & & \multirow{2}{*}{ Salt-N } & $25 \%$ ETm & \multirow{2}{*}{\multicolumn{2}{|c|}{$\begin{array}{l}11.0 \pm 0.58 \\
27.1 \pm 2.54\end{array}$}} & \multirow{2}{*}{\multicolumn{2}{|c|}{$\begin{array}{l}0.00 \pm 0.00 \\
12.5 \pm 2.39\end{array}$}} & \multirow{2}{*}{\multicolumn{2}{|c|}{$\begin{array}{l}1.63 \pm 0.63 \\
5.00 \pm 0.27\end{array}$}} \\
\hline & & & $100 \%$ ETm & & & & & & \\
\hline \multirow{8}{*}{ Unikum } & \multirow{4}{*}{ AMF-Y } & \multirow{2}{*}{ Salt-Y } & $25 \%$ ETm & \multirow{2}{*}{\multicolumn{2}{|c|}{$\begin{array}{l}15.5 \pm 1.39 \\
30.5 \pm 1.79\end{array}$}} & \multirow{2}{*}{\multicolumn{2}{|c|}{$\begin{array}{l}0.00 \pm 0.00 \\
16.0 \pm 1.27\end{array}$}} & 4.38 & 0.18 \\
\hline & & & $100 \%$ ETm & & & & & 4.75 & 0.25 \\
\hline & & & $25 \%$ ETm & 17.1 & 2.79 & 9.81 & 2.28 & 4.25 & 0.25 \\
\hline & & Salt-N & $100 \%$ ETm & 47.4 & 1.68 & 21.2 & 0.87 & 4.75 & 0.25 \\
\hline & & & $25 \%$ ETm & 17.5 & 2.44 & 0.00 & 0.00 & 4.00 & 0.38 \\
\hline & AMF-N & Salt-Y & $100 \%$ ETm & 33.6 & 0.86 & 17.3 & 0.60 & 5.13 & 0.13 \\
\hline & & & $25 \%$ ETm & 12.0 & 0.92 & 0.00 & 0.00 & 4.38 & 0.18 \\
\hline & & Salt-N & $100 \%$ ETm & 31.1 & 0.95 & 13.7 & 1.15 & 5.25 & 0.16 \\
\hline & ANC & VA & & Sign. & LSD & Sign. & LSD & Sign. & LSD \\
\hline & Genoty & e (G) & & $* *$ & 2.476 & $* *$ & 1.410 & * & 0.314 \\
\hline & corrhizal in & culation & & $* *$ & 2.476 & $* *$ & 1.410 & ns & - \\
\hline & Water sal & nity (S) & & $* *$ & 2.476 & $* *$ & 1.410 & $*$ & 0.314 \\
\hline & Water resti & ition (W) & & $* *$ & 2.476 & $* *$ & 1.410 & $* *$ & 0.416 \\
\hline & $\mathrm{G} \times$ & & & * & 2.647 & ns & - & $* *$ & 0.588 \\
\hline & $\mathbf{G}>$ & & & $* *$ & 3.502 & ns & - & ns & - \\
\hline & $\mathrm{G} \times$ & & & $* *$ & 3.502 & $* *$ & 1.994 & $* *$ & 0.588 \\
\hline & $\mathbf{M}>$ & & & $* *$ & 3.502 & $* *$ & 1.994 & ns & - \\
\hline & $\mathbf{M} \times$ & & & $* *$ & 3.502 & $* *$ & 1.994 & $* *$ & 0.588 \\
\hline & $\mathbf{S} \times$ & & & $* *$ & 3.502 & $* *$ & 1.994 & ns & - \\
\hline & $G \times N$ & $\times S$ & & ns & - & ns & - & ns & - \\
\hline & $\mathbf{G} \times \mathbf{N}$ & $\times \mathbf{W}$ & & ns & - & * & 2.820 & ns & - \\
\hline & $\mathrm{G} \times \mathrm{S}$ & $\times \mathbf{W}$ & & ns & - & ns & - & ns & - \\
\hline & $\mathbf{M} \times \mathbf{S}$ & $\times \mathbf{W}$ & & ns & - & $*$ & 2.820 & ns & - \\
\hline & $\mathbf{G} \times \mathbf{M}>$ & $\mathbf{S} \times \mathbf{W}$ & & ns & - & ns & - & ns & - \\
\hline
\end{tabular}

Unikum showed a significantly $(p<0.05)$ greater culm height $(+23.6 \%)$ than Kinelskoje $(20.7 \pm 1.5 \mathrm{~cm})$. Only in Kinelskoje did AMF inoculation result in a significant culm height increase 
$(+49.7 \%)$ as compared to the AMF-N treatment. Regardless of water restitution levels, in the Salt-N treatments, AMF inoculation significantly $(p<0.05)$ increased culm height $(+54.6 \%)$, whereas in the Salt-Y treatments, no differences were detected between AMF-Y and AMF-N (grand mean $=20.5 \pm 1.6 \mathrm{~cm}$ ). Salt stress resulted in a significant $(p<0.05)$ culm height decrease in Kinelskoje $(-32.6 \%)$ compared to the Salt-N treatment $(24.7 \pm 2.5 \mathrm{~cm})$, whereas no difference was observed in Unikum $(25.6 \pm 2.1 \mathrm{~cm}$ on average). At $100 \%$ ETm water restitution, AMF inoculation resulted in a significantly $(p<0.05)$ greater culm height than the uninoculated treatments, whereas at 25\% ETm water restitution, AMF inoculation did not signify a significant effect on culm height $(14.6 \pm 1.1 \mathrm{~cm}$ on average). Considering the interaction between water restitution and genotype, Unikum showed a significantly $(p<0.05)$ higher culm height $(+28.3 \%)$ than Kinelskoje $(27.8 \pm 2.0 \mathrm{~cm})$ at $100 \%$ ETm water restitution, whereas no difference was found at $25 \%$ ETm water restitution ( $14.6 \pm 1.1 \mathrm{~cm}$ on average).

The treatments effect on culm fresh weight is reported in Table 4 . The two genotypes did not show significant differences in culm fresh weight (grand mean $=1.2 \pm 0.1 \mathrm{~g} \mathrm{plant}^{-1}$ ). Salinity stress, significantly affected the culm fresh weight which was lowered by $-39.5 \%$ ( $25 \% \mathrm{ETm})$ and $-42.5 \%$ $(100 \% \mathrm{ETm})$ as compared to the Salt-N treatments. At full ETm restitution and in absence of salt treatment, AMF inoculation determined a significant $(p<0.05)$ culm fresh weight increase $(+86.8 \%)$ compared to the un-inoculated control whereas under salinity stress, no AMF effect was detected. No significant response to AMF inoculation was observed at $25 \%$ ETm restitution whatever the irrigation water salinity.

Table 4. Proso millet fresh weight yield components.

\begin{tabular}{|c|c|c|c|c|c|c|c|c|c|}
\hline \multicolumn{4}{|c|}{ Treatments } & \multicolumn{2}{|c|}{ Culm (g) } & \multicolumn{2}{|c|}{ Panicle (g) } & \multicolumn{2}{|c|}{ Leaves (g) } \\
\hline \multirow{8}{*}{ Kinelskoje } & \multirow{4}{*}{ AMF-Y } & \multirow{2}{*}{ Salt-Y } & 25\% ETm & \multirow{2}{*}{\multicolumn{2}{|c|}{$\begin{array}{l}0.37 \pm 0.11 \\
1.36 \pm 0.29\end{array}$}} & \multirow{2}{*}{\multicolumn{2}{|c|}{$\begin{array}{l}0.16 \pm 0.11 \\
0.42 \pm 0.11\end{array}$}} & \multirow{2}{*}{\multicolumn{2}{|c|}{$\begin{array}{l}0.63 \pm 0.23 \\
1.10 \pm 0.16\end{array}$}} \\
\hline & & & $100 \%$ ETm & & & & & & \\
\hline & & \multirow{2}{*}{ Salt-N } & 25\% ETm & \multirow{2}{*}{\multicolumn{2}{|c|}{$\begin{array}{l}0.96 \pm 0.17 \\
2.82 \pm 0.24\end{array}$}} & \multirow{2}{*}{\multicolumn{2}{|c|}{$\begin{array}{l}0.47 \pm 0.07 \\
0.57 \pm 0.06\end{array}$}} & \multirow{2}{*}{\multicolumn{2}{|c|}{$\begin{array}{l}0.93 \pm 0.08 \\
1.13 \pm 0.15\end{array}$}} \\
\hline & & & $100 \%$ ETm & & & & & & \\
\hline & \multirow{4}{*}{ AMF-N } & \multirow[b]{2}{*}{ Salt-Y } & 25\% ETm & \multirow{2}{*}{\multicolumn{2}{|c|}{$\begin{array}{l}0.22 \pm 0.04 \\
0.81 \pm 0.09\end{array}$}} & \multirow{2}{*}{\multicolumn{2}{|c|}{$\begin{array}{l}0.00 \pm 0.00 \\
0.54 \pm 0.08\end{array}$}} & \multirow{2}{*}{\multicolumn{2}{|c|}{$\begin{array}{l}0.22 \pm 0.04 \\
0.67 \pm 0.12\end{array}$}} \\
\hline & & & $100 \%$ ETm & & & & & & \\
\hline & & \multirow{2}{*}{ Salt-N } & 25\% ETm & \multirow{2}{*}{\multicolumn{2}{|c|}{$\begin{array}{l}0.34 \pm 0.04 \\
1.74 \pm 0.27\end{array}$}} & \multirow{2}{*}{\multicolumn{2}{|c|}{$\begin{array}{l}0.00 \pm 0.00 \\
0.59 \pm 0.11\end{array}$}} & \multirow{2}{*}{\multicolumn{2}{|c|}{$\begin{array}{l}0.15 \pm 0.08 \\
1.10 \pm 0.18\end{array}$}} \\
\hline & & & $100 \%$ ETm & & & & & & \\
\hline \multirow{8}{*}{ Unikum } & \multirow{4}{*}{ AMF-Y } & \multirow{2}{*}{ Salt-Y } & 25\% ETm & \multirow{2}{*}{\multicolumn{2}{|c|}{$\begin{array}{l}0.41 \pm 0.18 \\
1.69 \pm 0.29\end{array}$}} & \multirow{2}{*}{\multicolumn{2}{|c|}{$\begin{array}{l}0.00 \pm 0.00 \\
0.84 \pm 0.15\end{array}$}} & 0.58 & 0.17 \\
\hline & & & $100 \%$ ETm & & & & & 1.04 & 0.23 \\
\hline & & & 25\% ETm & 0.54 & 0.12 & 0.53 & 0.11 & 0.79 & 0.06 \\
\hline & & Salt-N & $100 \%$ ETm & 3.38 & 0.44 & 1.12 & 0.16 & 1.40 & 0.12 \\
\hline & & & 25\% ETm & 0.40 & 0.07 & 0.00 & 0.00 & 0.61 & 0.23 \\
\hline & AMF-N & Salt-Y & $100 \%$ ETm & 1.61 & 0.20 & 0.94 & 0.25 & 1.18 & 0.15 \\
\hline & & & 25\% ETm & 0.47 & 0.10 & 0.00 & 0.00 & 0.47 & 0.10 \\
\hline & & Salt-N & $100 \%$ ETm & 1.58 & 0.22 & 0.85 & 0.17 & 1.34 & 0.11 \\
\hline & AN & VA & & Sign. & LSD & Sign. & LSD & Sign. & LSD \\
\hline & Geno & pe (G) & & ns & - & $* *$ & 0.148 & * & 0.140 \\
\hline & corrhizal & oculation & & $* *$ & 0.249 & $* *$ & 0.148 & * & 0.140 \\
\hline & Water s & inity (S) & & $* *$ & 0.249 & $* *$ & 0.148 & $* *$ & 0.185 \\
\hline & Water res & ution (W & & $* *$ & 0.249 & $* *$ & 0.148 & $* *$ & 0.185 \\
\hline & & $\mathbf{M}$ & & ns & - & ns & - & * & 0.198 \\
\hline & & $\mathrm{S}$ & & ns & - & ns & - & ns & - \\
\hline & & W & & * & 0.266 & $* *$ & 0.210 & ns & - \\
\hline & & $S$ & & $* *$ & 0.352 & $* *$ & 0.210 & ns & - \\
\hline & $\mathbf{M}$ & $\mathbf{W}$ & & $* *$ & 0.352 & * & 0.159 & ns & - \\
\hline & & W & & $* *$ & 0.352 & ns & - & ns & - \\
\hline & $G \times$ & $I \times S$ & & ns & - & ns & - & ns & - \\
\hline & $G \times$ & $\times \mathbf{W}$ & & ns & - & ns & - & ns & - \\
\hline & $G \times$ & $\times \mathbf{W}$ & & ns & - & ns & - & ns & - \\
\hline & $\mathbf{M} \times$ & $\times \mathbf{W}$ & & ns & - & ns & - & ns & - \\
\hline & $\mathbf{G} \times \mathbf{M}$ & $\mathbf{S} \times \mathbf{W}$ & & ns & - & ns & - & ns & - \\
\hline
\end{tabular}

${ }^{*}=p<0.05 ;{ }^{* *}=p<0.01 ; \mathrm{ns}=$ not significant. 


\subsubsection{Panicle Length and Fresh Panicle Weight}

The treatment effects on panicle length are reported in Table 3. In the absence of salt at both water restitution levels, significant positive effects $(p<0.05)$ of AMF inoculation were observed on panicle length. In presence of the salt stress, AMF inoculation did not result in significant differences in panicle length, with grand mean values of $0.5 \pm 0.4 \mathrm{~cm}(25 \% \mathrm{ETm})$ and $13.7 \pm 1.0 \mathrm{~cm}(100 \% \mathrm{ETm})$. However, the panicle growth was completely inhibited by the lowest water restitution (25\% ETm) in AMF-N treatment. At $100 \%$ ETm water restitution, Unikum showed a panicle length significantly $(p<0.05)$ higher $(+43.0 \%)$ than Kinelskoje $(11.9 \pm 0.7 \mathrm{~cm})$, whereas no difference in this parameter was observed at $25 \% \mathrm{ETm}(2.9 \pm 1.0 \mathrm{~cm}$ on average $)$.

The treatment effects on panicle fresh weight are reported in Table 4. Here, 100\% ETm AMF inoculation did not exert a positive effect on panicle fresh weight, since no difference was observed between the two AMF treatments, with a grand mean value of $0.7 \pm 0.1 \mathrm{~g} \mathrm{plant}^{-1}$. Comparing genotypes, the $100 \%$ ETm water restitution resulted in a significantly $(p<0.05)$ higher panicle fresh weight $(+77.2 \%)$ in Unikum than Kinelskoje $\left(0.5 \pm 0.1 \mathrm{~g} \mathrm{plant}^{-1}\right)$. No differences were found between the two millet genotypes at the lowest water restitution $(25 \%$ ETm, with a grand mean $0.2 \pm 0.1 \mathrm{~g}$ plant $\left.^{-1}\right)$. Regardless of the other factor levels, Unikum showed a significantly $(p<0.05)$ higher panicle fresh weight $(+53.6 \%)$ than Kinelskoje $\left(0.4 \pm 0.1 \mathrm{~g} \mathrm{plant}^{-1}\right)$. Salt- $Y$ treatment negatively affected the panicle fresh weight, with a significant $(p<0.05)$ decrease $(-29.9 \%)$ compared to the Salt-N treatment $\left(0.5 \pm 0.1 \mathrm{~g} \mathrm{plant}^{-1}\right)$. AMF inoculation resulted in a significantly $(p<0.05)$ higher panicle fresh weight $(+41.5 \%)$ than uninoculated treatment $\left(0.4 \pm 0.1 \mathrm{~g} \mathrm{plant}^{-1}\right)$.

\subsubsection{Leaf Number and Leaf Fresh Weight}

The treatment effects on leaf number and leaf fresh weight are reported in Tables 3 and 4, respectively. Leaf number was significantly $(p<0.05)$ higher in Unikum $(+9.7 \%)$ as compared to Kinelskoje $\left(4.2 \pm 0.2\right.$ leaves culm $\left.{ }^{-1}\right)$. In Kinelskosje, AMF inoculation resulted in a significant $(p<0.05)$ increase in leaf number $(+24.2 \%)$ and fresh weight $(+76.2 \%)$ compared to the uninoculated plants (3.8 \pm 0.3 leaves culm ${ }^{-1}$ and $\left.0.5 \pm 0.1 \mathrm{~g} \mathrm{culm}^{-1}\right)$, whereas AMF inoculation did not show any effects in Unikum ( $4.6 \pm 0.1$ leaves culm ${ }^{-1}$ and $\left.0.9 \pm 0.1 \mathrm{~g} \mathrm{culm}^{-1}\right)$. Regardless of the millet genotype, at $100 \%$ ETm restitution and despite the salt treatments, AMF inoculation did not affect leaf numbers, whereas at $25 \%$ ETm restitution, a positive $(p<0.05)$ effect of AMF inoculation was observed in the absence of salt stress.

The two genotypes, at 100\% ETm water restitution, did not show significant differences in the number of leaves. On the contrary, at the lowest water restitution level (25\% ETm), a significant $(p<0.01)$ reduction in the number of leaves was observed in both genotypes. In particular, Kineslkosje showed a decrease $(-19.8 \%)$ compared to the Unikum $\left(4.3 \pm 0.1\right.$ leaves culm $\left.{ }^{-1}\right)$.

Considering the stresses, salt irrigation and water restitution resulted in a significant $(p<0.01)$ fresh weight decrease in leaves with Salt-Y $(-17.7 \%)$ and $25 \%$ ETm $(-51.1 \%)$ as compared to Salt-N $\left(0.9 \pm 0.04 \mathrm{~g} \mathrm{culm}^{-1}\right)$ and $100 \%$ ETm water restitution $\left(1.1 \pm 0.04 \mathrm{~g} \mathrm{culm}^{-1}\right)$.

\subsubsection{Leaf Chlorophyll Content (SPAD index)}

AMF inoculation resulted in a significant $(p<0.01)$ increase $(+13.7 \%)$ in leaf SPAD values compared to the AMF-N treatment $(37.8 \pm 1.2)$ in Kinelskoje, but not in Unikum (grand mean SPAD value $=$ $40.9 \pm 0.8$ ) (Figure 7a). At 25\% ETm restitution, Kinelskoje showed a significant $(p<0.01)$ decrease $(-18.1 \%)$ in leaf SPAD value as compared to full water restitution $(44.4 \pm 0.5)$, whereas no statistical difference was found in Unikum, with an average leaf SPAD value of $40.9 \pm 0.8$ (Figure 7b). No effect was found due to irrigation water salinity levels, regardless of other studied factors. 

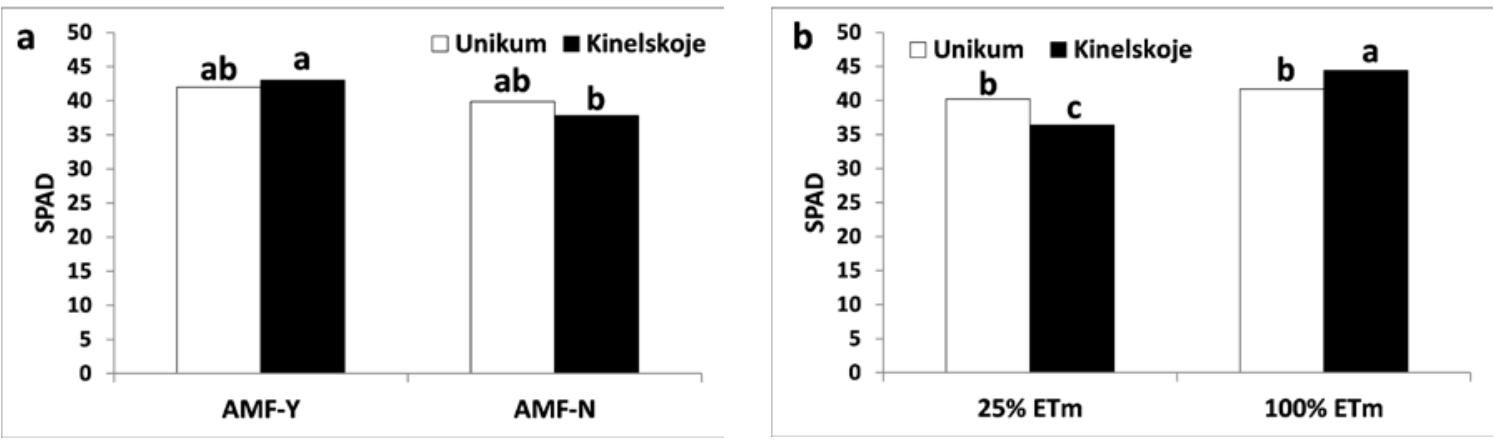

Figure 7. Effect of the studied factors on the SPAD value. (a) Genotype X AMF inoculation; (b) genotype $X$ ETm restitution. Different letters show statistical differences of the treatments at $p<0.01$ (Least Significant Difference-Fisher test). AMF-Y = AMF-inoculated treatment; AMF-N = AMF uninoculated treatment; $25 \%$ ETm = restitution of crop evapotranspirated water at $25 \% ; 100 \%$ ETm = restitution of crop evapotranspirated water at $100 \%$.

\subsubsection{Leaf Area Surface}

Salt treatment did not result in any different leaf area surface in the plants present in the AMF-N plots. The AMF inoculation resulted in a significant $(p<0.05)$ increase $(+80.5 \%)$ in leaf area surface with Salt-N treatment as compared to Salt-Y treatment $\left(26.6 \pm 5.3 \mathrm{~cm}^{2}\right.$ plant $\left.^{-1}\right)$ (Figure 8a). Regardless of the genotypes, in the absence of AMF inoculation, at $25 \%$ ETm water restitution we observed a significant $(p<0.05)$ decrease in leaf area surface $(-68.1 \%)$ compared to $100 \%$ ETm restitution $\left(47.2 \pm 3.7 \mathrm{~cm}^{2}\right.$ plant $^{-1}$ ). No significant difference was found in presence of AMF inoculation (Figure $8 b$ ) with either of the water restitution levels.
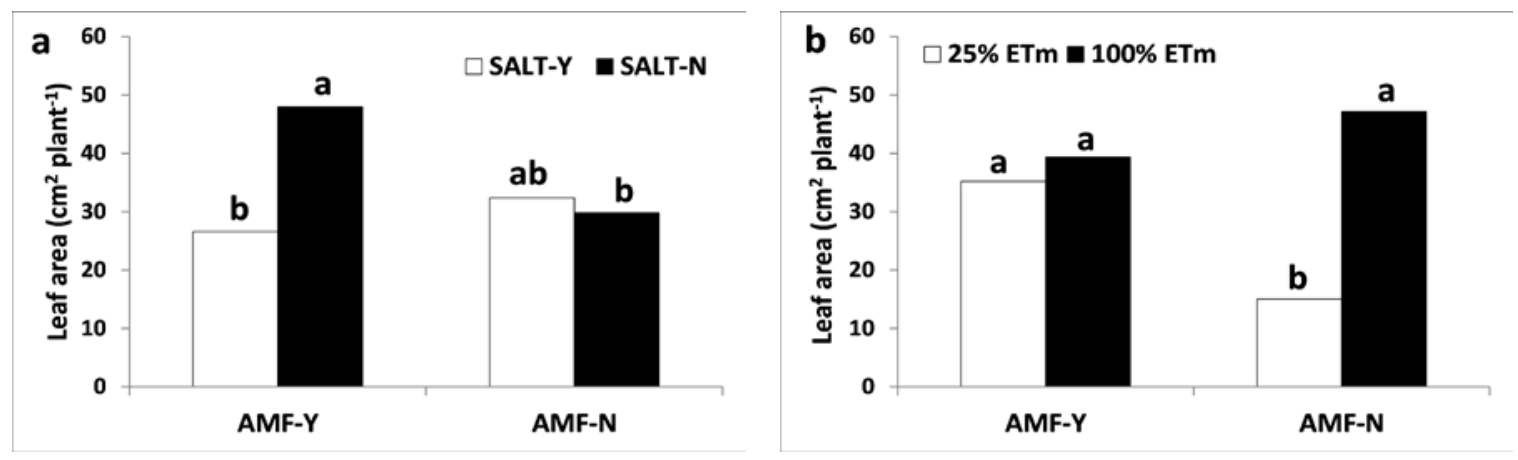

Figure 8. Effect of the studied factors on leaf area surface. (a) Salt X AMF inoculation; (b) ETm restitution X AMF inoculation. Different letters show statistical differences of the treatments at $p<0.05$ (Least Significant Difference-Fisher test). AMF-Y = AMF-inoculated treatment; AMF-N = AMF uninoculated treatment; Salt- $\mathrm{Y}=$ treatment with $\mathrm{NaCl}$ addition in irrigation water; Salt- $\mathrm{N}$ = treatment without $\mathrm{NaCl}$ addition in irrigation water; $25 \% \mathrm{ETm}$ = restitution of crop evapotranspirated water at $25 \% ; 100 \%$ ETm $=$ restitution of crop evapotranspirated water at $100 \%$.

\section{Discussion}

\subsection{Laboratory Experiment}

In Mediterranean areas, the germination process is affected by abiotic stresses such as high salinity and drought, which results in a lower seed water absorption [47] and affects the mobilization of stored reserves or the synthesis of proteins directly in germinating embryos [48], thus jeopardizing growth processes. 
Almansouri et al. [48], in a study on durum wheat, showed a reduction or inhibition in germination in response to a decrease in water potential. However, these authors, at an osmotic pressure similar to the one tested in our study $(-0.580 \mathrm{MPa})$, did not find any effect on final germination percentage in response to iso-osmotic solution of $\mathrm{NaCl}$ and mannitol. In our case, seed water uptake was negatively affected by the external water potential decrease due to the reduced diffusivity of water to the seed coats, but the seed moisture was sufficient to ensure good germination in all genotypes.

Generally, seed germination decreased with increasing salt concentrations and salt-specific effects $[49,50]$. In our study, the three different genotypes were not affected by the levels of salt applied, showing tolerance to high salinity. Sabir and Ashraf [51], with 18 millet accessions collected from different areas of Pakistan, demonstrated an inter-cultivar variation for $\mathrm{NaCl}$ tolerance $(0.0,-0.300$, -0.600 and $-0.900 \mathrm{MPa}$ ). These authors reported a general significant salt-induced depression on seed germination in all the accessions, except for the four which showed the highest percentages at $-0.900 \mathrm{MPa}$. In our experiment, although salt stress range was more limited (up to $-0.750 \mathrm{MPa}$ ) as compared to the Sabir and Ashraf [51] study, the three genotypes were not affected by salt levels, suggesting salt tolerance. The lack of differences in seed water uptake, germination, and seedling vigor index obtained in our study comparing osmotica ( $\mathrm{NaCl}$ or mannitol) can be explained by a similar penetrating behavior of $\mathrm{NaCl}$ and mannitol in the plant tissue that contributes to adjusting the internal osmotic potential decrease in the germinating seeds, thus allowing sufficient water uptake to be maintained under a high external water potential.

\subsection{Field Experiment}

The FBY showed genotype and salinity-specific responses indicating Unikum, among the studied genotypes, as the best-performing genotype under salinity and water stress conditions. Particularly, without AMF inoculation, Unikum did not show different FBY on comparing the two studied salt stress levels, suggesting that this genotype is more adept under saline conditions. In addition, Unikum was the genotype that took advantage of the AMF inoculation in the absence of saline stress, suggesting that the use of biofertilizers can be a valid agronomic practice to maximize fodder production when this genotype is used. In presence of salt treatment, regardless of the genotype, the effect of AMF inoculation was inhibited, resulting in the mycorrhizal inoculum not promoting the FBY. This finding is not in line with that of Daei et al. [52] who reported, in durum wheat cultivated under saline conditions $\left(7.41 \mathrm{dS} \mathrm{m}^{-1}\right)$, a significant increase on growth and grain yield of mycorrhized plants due to enhanced nutrient uptake. Juniper and Abbott [7] showed that the germination of spores of all the AMF fungi tested was delayed and the hyphal growth from propagules was reduced in the presence of $\mathrm{NaCl}$. This latter result could explain the absence of a significant AMF effect on FBY at the end of crop cycle. AMF inoculation resulted in a better FBY response under water stress conditions, whereas no effects were seen in well-watered plots. This finding is in agreement with the results obtained by Porcel et al. [53] and Saia et al. [54] in different pedo-climatic conditions on sorghum and berseem clover. They reported a positive AMF effect under water shortage and no AMF effect at full ETm restitution. At 100\% ETm restitution, AMF inoculation significantly decreased shoot density and increased culm height. In contrast, under water stress conditions (25\% ETm) AMF inoculation increased shoot density but did not influence culm height. These results suggest that: (1) under water stress conditions ( $25 \% \mathrm{ETm})$, in the presence of AMF inoculation the increase in FBY can be attributed to higher shoot density; and (2) under well-watered conditions (100\% ETm), the absence of a significant difference in the FBY between AMF treatments can be attributed to an increase of mycorrhized plant weight despite the lower shoot density. Considering the panicle emission and development, a positive effect was exerted by AMF inoculation in both ETm restitution levels, especially in the absence of salt stress, suggesting that the mycorrhizal fungi promoted the transition from a vegetative to a reproductive phenological phase (Oladele and Awodun 2014) [55]. All experimental factors exerted significant influences on leaf characteristics. AMF inoculation promoted a positive effect on leaf size and fresh and dry weight (data not shown), in agreement with Augé et al. (2015) [56] and Fagbola et al. (2001) [57] which reported 
respectively an improved leaf water status and leaf dry weight in mycorrhized plants. Considering the SPAD value, AMF inoculation increased this parameter, confirming the mycorrhizal positive effect on plant physiological activity (Zhou et al. 2015) [58].

Saline water irrigation negatively influenced the leaf fresh weight, without significant effects on leaf number, dry weight, and surface, supporting that salt stress mainly reduces leaf water content (Fidalgo et al. 2004) [59]. Moreover, salinity stress did not exert any significant effects on SPAD values in either of genotypes, despite this stress resulting in chlorophyll damage, with a reduction in photosynthetic activity and leaf senescence acceleration (Shi and Guo, 2006; Beltrano et al. 2013) [60,61]. No reduction in leaf chlorophyll content was observed, probably due to the relatively brief exposition to salt stress because of the short growing season. Water stress conditions ( $25 \% \mathrm{ETm})$ exerted highly negative effects on leaf characteristics; in these conditions, AMF inoculation could partially overcome such negative effects, confirming their beneficial role in plant water status under drought stress (Beltrano and Ronco 2008; Grümberg et al. 2015) [62,63].

\section{Conclusions}

Osmotic stress resulted in a genotype-specific response, with the strongest reduction in seed water uptake, germination, and seedling vigor index observed at $-0.750 \mathrm{MPa}$. Unikum germination (95.3\%) was not affected by osmotic pressures with a higher SVI as compared to Kinelskoje and VIR 9181. Unikum was the best genotype in the laboratory trial, and this finding was also confirmed in open field conditions producing more than double the FBY of Kinelskoje. Although the AMF inoculation effect was negatively influenced by irrigation with saline water, it exercised a positive effect $(+377.3 \%)$ on FBY under water stress. The present study gives novel information about proso millet forage production, highlighting the potential role of AMF in bio-fertilizers in low-input sustainable agriculture in marginal, semi-arid Mediterranean land, but further large-scale studies are needed to confirm our findings.

Acknowledgments: The authors would like to thank Centro Ricerche e Sperimentazione per il miglioramento vegetale (CERMIS) - (Italy) for providing us with the proso millet genotypes, and Valeria Cavallaro for providing helpful feedback on a previous draft of this manuscript. Meteorological data were provided by the Agro-Meteorological Information Service of Sicily (SIAS).

Author Contributions: C.C., C.M., and A.C.B. conceived and designed the experiments; C.C., C.M., and A.C.B. performed the experiments; C.C., A.B, C.M., and A.C.B. analyzed the data; A.C.B. contributed reagents/materials/analysis tools; C.C., A.B, C.M., M.B., and A.C.B. wrote the paper.

Conflicts of Interest: The authors declare no conflict of interest.

\section{References}

1. Hasegawa, P.M.; Bressan, R.A.; Zhu, J.K.; Bohnert, H.J. Plant cellular and molecular responses to high salinity. Annu. Rev. Plant Biol. 2000, 51, 463-499. [CrossRef] [PubMed]

2. Zhu, J.K. Regulation of ion homeostasis under salt stress. Curr. Opin. Plant Biol. 2003, 6, 441-445. [CrossRef]

3. Mahajan, S.; Tuteja, N. Cold, salinity and drought stresses: An overview. Arch. Biochem. Biophys. 2005, 444, 139-158. [CrossRef] [PubMed]

4. Villa-Castorena, M.; Ulery, A.L.; Catalán-Valencia, E.A.; Remmenga, M.D. Salinity and nitrogen rate effects on the growth and yield of chile pepper plants. Soil Sci. Soc. Am. J. 2003, 67, 1781-1789. [CrossRef]

5. Al-Karaki, G.N. Nursery inoculation of tomato with arbuscular mycorrhizal fungi and subsequent performance under irrigation with saline water. Sci. Hortic. 2006, 109, 1-7. [CrossRef]

6. Jones, A.; Panagos, P.; Barcelo, S.; Bouraoui, F.; Bosco, C.; Dewitte, O.; Gardi, C.; Hervás, J.; Hiederer, R.; Jeffery, S.; et al. The State of Soil in Europe-A Contribution of the JRC to the European Environment Agency's Environment State and Outlook Report-SOER 2010; Publications Office of the European Union: Luxembourg, 2012.

7. Juniper, S.; Abbott, L. Vesicular-arbuscular mycorrhizas and soil salinity. Mycorrhiza 1993, 4, 45-57. [CrossRef]

8. Ramoliya, P.J.; Patel, H.M.; Pandey, A.N. Effect of salinization of soil on growth and macro-and micro-nutrient accumulation in seedlings of Salvadora persica (Salvadoraceae). For. Ecol. Manag. 2004, 202, 181-193. [CrossRef] 
9. Aggarwal, A.; Kadian, N.; Neetu, K.; Tanwar, A.; Gupta, K.K. Arbuscular mycorrhizal symbiosis and alleviation of salinity stress. J. Appl. Nat. Sci. 2012, 4, 144-155.

10. Adiku, S.G.K.; Renger, M.; Wessolek, G.; Facklam, M.; Hecht-Bucholtz, C. Simulation of the dry matter production and seed yield of common beans under varying soil water and salinity conditions. Agric. Water Manag. 2001, 47, 55-68. [CrossRef]

11. Marschner, H. Mineral Nutrition of Higher Plants, 2nd ed.; Academic Press: Cambridge, MA, USA, 1995.

12. Ipsilantis, I.; Karpouzas, D.G.; Papadopoulou, K.K.; Ehaliotis, C. Effects of soil application of olive mill wastewaters on the structure and function of the community of arbuscular mycorrhizal fungi. Soil Biol. Biochem. 2009, 41, 2466-2476. [CrossRef]

13. Candido, V.; Campanelli, G.; D'Addabbo, T.; Castronuovo, D.; Renco, M.; Camele, I. Growth and yield promoting effect of artificial mycorrhization combined with different fertiliser rates on field-grown tomato. Ital. J. Agron. 2013, 8, 168-174. [CrossRef]

14. Sabia, E.; Claps, S.; Morone, G.; Bruno, A.; Sepe, L.; Aleandri, R. Field inoculation of arbuscular mycorrhiza on maize (Zea mays L.) under low inputs: Preliminary study on quantitative and qualitative aspects. Ital. J. Agron. 2015, 10, 30-33. [CrossRef]

15. Tarraf, W.; Ruta, C.; De Cillis, F.; Tagarelli, A.; Tedone, L.; De Mastro, G. Effects of mycorrhiza on growth and essential oil production in selected aromatic plants. Ital. J. Agron. 2015, 10, 160-162. [CrossRef]

16. Puia, C.; Vidican, R.; Szabó, G.; Stoian, V. Potential of biofertilisers to improve performance of local genotype tomatoes. Ital. J. Agron. 2017, 12, 192-200. [CrossRef]

17. Ruiz-Lozano, J.M. Arbuscular mycorrhizal symbiosis and alleviation of osmotic stress. New perspectives for molecular studies. Mycorrhiza 2003, 13, 309-317. [CrossRef] [PubMed]

18. Evelin, H.; Kapoor, R.; Giri, B. Arbuscular mycorrhizal fungi in alleviation of salt stress: A review. Ann. Bot. 2009, 104, 1263-1280. [CrossRef] [PubMed]

19. Miransari, M. Contribution of arbuscular mycorrhizal symbiosis to plant growth under different types of soil stress. Plant Biol. 2010, 12, 563-569. [CrossRef] [PubMed]

20. Porcel, R.; Aroca, R.; Ruiz-Lozano, J.M. Salinity stress alleviation using arbuscular mycorrhizal fungi. A review. Agron. Sustain. Dev. 2012, 32, 181-200. [CrossRef]

21. Langeroodi, A.R.S.; Ghooshchi, F.; Dadgar, T. Alleviatory activities in mycorrhizal tobacco plants subjected to increasing chloride in irrigation water. Ital. J. Agron. 2017. [CrossRef]

22. Wu, Q.S.; Zou, Y.N.; He, X.H. Contributions of arbuscular mycorrhizal fungi to growth, photosynthesis, root morphology and ionic balance of citrus seedlings under salt stress. Acta Physiol. Plant. 2010, 32, 297-304. [CrossRef]

23. Al-Karaki, G.N. Growth of mycorrhizal tomato and mineral acquisition under salt stress. Mycorrhiza 2000, 10, 51-54. [CrossRef]

24. Al-Karaki, G.N.; Hammad, R.; Rusan, M. Response of two tomato cultivars differing in salt tolerance to inoculation with mycorrhizal fungi under salt stress. Mycorrhiza 2001, 11, 43-47. [CrossRef]

25. Asghari, H.R.; Marschner, P.; Smith, S.E.; Smith, F.A. Growth response of Atriplex nummularia to inoculation with arbuscular mycorrhizal fungi at different salinity levels. Plant Soil 2005, 273, 245-256. [CrossRef]

26. Feng, G.; Zhang, F.; Li, X.; Tian, C.; Tang, C.; Rengel, Z. Improved tolerance of maize plants to salt stress by arbuscular mycorrhiza is related to higher accumulation of soluble sugars in roots. Mycorrhiza 2002, 12, 185-190. [PubMed]

27. Giri, B.; Kapoor, R.; Mukerji, K.G. Improved tolerance of Acacia nilotica to salt stress by arbuscular mycorrhiza, Glomus fasciculatum may be partly related to elevated $\mathrm{K} / \mathrm{Na}$ ratios in root and shoot tissues. Microb. Ecol. 2007, 54, 753-760. [CrossRef] [PubMed]

28. Asghari, H.R. Vesicular-arbuscular (VA) mycorrhizae improve salinity tolerance in pre-inoculation subterranean clover (Trifolium subterraneum) seedlings. Int. J. Plant Prod. 2012, 2, 243-256.

29. He, Z.; He, C.; Zhang, Z.; Zou, Z.; Wang, H. Changes of antioxidative enzymes and cell membrane osmosis in tomato colonized by arbuscular mycorrhizae under $\mathrm{NaCl}$ stress. Colloids Surf. B. 2007, 59, 128-133. [CrossRef] [PubMed]

30. Tian, C.Y.; Feng, G.; Li, X.L.; Zhang, F.S. Different effects of arbuscular mycorrhizal fungal isolates from saline or non-saline soil on salinity tolerance of plants. Appl. Soil Ecol. 2004, 26, 143-148. [CrossRef] 
31. Sheng, M.; Tang, M.; Chen, H.; Yang, B.; Zhang, F.; Huang, Y. Influence of arbuscular mycorrhizae on photosynthesis and water status of maize plants under salt stress. Mycorrhiza 2008, 18, 287-296. [CrossRef] [PubMed]

32. Juniper, S.; Abbott, L.K. Soil salinity delays germination and limits growth of hyphae from propagules of arbuscular mycorrhizal fungi. Mycorrhiza 2006, 16, 371-379. [CrossRef] [PubMed]

33. Upadhyaya, H.D.; Sharma, S.; Gowda, C.L.L.; Reddy, V.G.; Singh, S. Developing proso millet (Panicum miliaceum L.) core collection using geographic and morpho-agronomic data. Crop Pasture Sci. 2011, 62, 383-389. [CrossRef]

34. Channabasava, A.; Lakshman, H.C.; Jorquera, M.A. Effect of fungicides on association of arbuscular mycorrhiza fungus Rhizophagus fasciculatus and growth of proso millet (Panicum miliaceum L.). J. Soil Sci. Plant Nutr. 2015, 15, 35-45. [CrossRef]

35. Arab, A.; Bradaran, R.; Vahidipour, T.H. Effect of irrigation and mycorrhizal bio-fertilizers on yield and agronomic traits of millet (Panicum miliaceum L.). Int. J. Agri. Crop Sci. 2013, 6, 103-109.

36. Ledér, I. Sorghum and millet in cultivated plants, primarily as food sources. In Encyclopedia of Life Support Systems (EOLSS), Developed under the Auspices of the UNESCO; Eolss Publishers: Oxford, UK, 2004.

37. Food and Agriculture Organization (FAO). FAOSTAT Statistical Database 2014; FAO: Rome, Italy, 2014.

38. Yue, H.; Wang, M.; Liu, S.; Du, X.; Song, W.; Nie, X. Transcriptome-wide identification and expression profiles of the WRKY transcription factor family in Broomcorn millet (Panicum miliaceum L.). BMC Genom. 2016, 17, 343. [CrossRef] [PubMed]

39. Hunt, H.V.; Campana, M.G.; Lawes, M.C.; Park, Y.J.; Bower, M.A.; Howe, C.J.; Jones, M.K. Genetic diversity and phylogeography of broomcorn millet (Panicum miliaceum L.) across Eurasia. Mol. Ecol. 2011, 20, 4756-4771. [CrossRef] [PubMed]

40. Saleh, A.S.; Zhang, Q.; Chen, J.; Shen, Q. Millet grains: Nutritional quality, processing, and potential health benefits. Compr. Rev. Food Sci. Food Saf. 2013, 12, 281-295. [CrossRef]

41. Morales, J.U.; Alatorre, J.A.H.; Nieto, C.A.R.; Becerra, J.F.C. Forage production and nutritional content of silage from three varieties of pearl millet (Pennisetum glaucum) harvested at two maturity stages. J. Anim. Plant Sci. 2015, 27, 4161-4169.

42. Jahansouz, M.R.; Afshar, R.K.; Heidari, H.; Hashemi, M. Evaluation of yield and quality of sorghum and millet as alternative forage crops to corn under normal and deficit irrigation regimes. Jordan J. Agric. Sci. 2014, 10, 699-715.

43. Mohajer, S.; Ghods, H.; Taha, R.M.; Talati, A. Effect of different harvest time on yield and forage quality of three varieties of common millet (Panicum miliaceum). Sci. Res. Essays 2012, 7, 3020-3025. [CrossRef]

44. Ali, S.A.; Idris, A.Y. Germination and seedling growth of pearl millet (Pennisetum glaucum L.) cultivars under salinity conditions. Int. J. Plant Res. 2015, 1, 1-5.

45. Allen, R.G.; Pereira, L.S.; Raes, D.; Smith, M. Single crop coefficient (KC). In Crop Evapotranspiration-Guidelines for Computing Crop Water Requirements; FAO Irrigation and Drainage Paper 56; Food and Agriculture Organization of the United Nations (FAO): Rome, Italy, 1998; ISBN 92-5-104219-5.

46. Hess, M.; Barralis, G.; Bleiholder, H.; Buhr, L.; Eggers, T.H.; Hack, H.; Stauss, R. Use of the extended $\mathrm{BBCH}$ scale-General for the descriptions of the growth stages of mono; and dicotyledonous weed species. Weed Res. 1997, 37, 433-441. [CrossRef]

47. Dodd, G.L.; Donovan, L.A. Water potential and ionic effects on germination and seedling growth of two cold desert shrubs. Am. J. Bot. 1999, 86, 1146-1153. [CrossRef] [PubMed]

48. Almansouri, M.; Kinet, J.M.; Lutts, S. Effect of salt and osmotic stresses on germination in durum wheat (Triticum durum Desf.). Plant Soil 2001, 231, 243-254. [CrossRef]

49. Ryan, J.; Miyamoto, S.; Stroehlein, J.L. Salt and specific ion effects on germination of four grass. J. Range Manag. 1975, 28, 61-64. [CrossRef]

50. Cavallaro, V.; Barbera, A.C.; Maucieri, C.; Gimma, G.; Scalisi, C.; Patanè, C. Evaluation of variability to drought and saline stress through the germination of different ecotypes of carob (Ceratonia siliqua L.) using a hydrotime model. Ecol. Eng. 2016, 95, 557-566. [CrossRef]

51. Sabir, P.; Ashraf, M. Inter-cultivar variation for salt tolerance in proso millet (Panicum miliaceum L.) at the germination stage. Pak. J. Bot. 2008, 40, 677-682. 
52. Daei, G.; Ardekani, M.R.; Rejali, F.; Teimuri, S.; Miransari, M. Alleviation of salinity stress on wheat yield, yield components, and nutrient uptake using arbuscular mycorrhizal fungi under field conditions. J. Plant Physiol. 2009, 166, 617-625. [CrossRef] [PubMed]

53. Porcel, R.; Ruiz-Lozano, J.M. Arbuscular mycorrhizal influence on leaf water potential, solute accumulation, and oxidative stress in soybean plants subjected to drought stress. J. Exp. Bot. 2004, 55, 1743-1750. [CrossRef] [PubMed]

54. Saia, S.; Amato, G.; Frenda, A.S.; Giambalvo, D.; Ruisi, P. Influence of arbuscular mycorrhizae on biomass production and nitrogen fixation of berseem clover plants subjected to water stress. PLoS ONE 2014, 9, e90738. [CrossRef] [PubMed]

55. Oladele, S.; Awodun, M. Response of lowland rice to biofertilizers inoculation and their effects on growth and yield in southwestern Nigeria. J. Agric. Environ. Sci. 2014, 3, 371-390.

56. Augé, R.M.; Toler, H.D.; Saxton, A.M. Arbuscular mycorrhizal symbiosis alters stomatal conductance of host plants more under drought than under amply watered conditions: A meta-analysis. Mycorrhiza 2015, 25, 13-24. [CrossRef] [PubMed]

57. Fagbola, O.; Osonubi, O.; Mulongoy, K.; Odunfa, S. Effects of drought stress and arbuscular mycorrhiza on the growth of Gliricidia sepium (Jacq). Walp, and Leucaena leucocephala (Lam.) de Wit. in simulated eroded soil conditions. Mycorrhiza 2001, 11, 215-223. [CrossRef]

58. Zhou, Q.; Ravnskov, S.; Jiang, D.; Wollenweber, B. Changes in carbon and nitrogen allocation, growth and grain yield induced by arbuscular mycorrhizal fungi in wheat (Triticum aestivum L.) subjected to a period of water deficit. Plant Growth Regul. 2015, 75, 751-760. [CrossRef]

59. Fidalgo, F.; Santos, A.; Santos, I.; Salema, R. Effects of long-term salt stress on antioxidant defense systems, leaf water relations and chloroplast ultrastructure of potato plants. Ann. Appl. Biol. 2004, 145, 185-192. [CrossRef]

60. Shi, L.X.; Guo, J.X. Changes in photosynthetic and growth characteristics of Leymus chinensis community along the retrogression on the Songnen grassland in northeastern China. Photosynthetica 2006, 44, 542-547. [CrossRef]

61. Beltrano, J.; Ronco, M.G. Improved tolerance of wheat plants (Triticum aestivum L.) to drought stress and rewatering by the arbuscular mycorrhizal fungus Glomus claroideum: Effect on growth and cell membrane stability. Braz. J. Plant Physiol. 2008, 20, 29-37. [CrossRef]

62. Beltrano, J.; Ruscitti, M.; Arango, M.C.; Ronco, M. Effects of arbuscular mycorrhiza inoculation on plant growth, biological and physiological parameters and mineral nutrition in pepper grown under different salinity and p levels. J. Soil Sci. Plant Nutr. 2013, 13, 123-141. [CrossRef]

63. Grümberg, B.C.; Urcelay, C.; Shroeder, M.A.; Vargas-Gil, S.; Luna, C.M. The role of inoculum identity in drought stress mitigation by arbuscular mycorrhizal fungi in soybean. Biol. Fertil. Soils 2015, 51, 1-10. [CrossRef]

(C) 2018 by the authors. Licensee MDPI, Basel, Switzerland. This article is an open access article distributed under the terms and conditions of the Creative Commons Attribution (CC BY) license (http://creativecommons.org/licenses/by/4.0/). 